\title{
A Parasite Coat Protein Binds Suramin to Confer Drug Resistance
}

Authors: Johan Zeelen ${ }^{1 *}$, Monique van Straaten ${ }^{1 *}$, Joseph Verdi ${ }^{1,2}$, Alexander Hempelmann ${ }^{1}$, Hamidreza Hashemi ${ }^{2}$, Kathryn Perez ${ }^{3}$, Philip D. Jeffrey ${ }^{4}$, Silvan Hälg ${ }^{5,6}$, Natalie Wiedemar ${ }^{5,6}$, Pascal Mäser ${ }^{5,6}$, F. Nina Papavasiliou ${ }^{2}$, C. Erec Stebbins ${ }^{1 \dagger}$

\section{Affiliations:}

${ }^{1}$ Division of Structural Biology of Infection and Immunity, German Cancer Research Center

${ }^{2}$ Division of Immune Diversity, German Cancer Research Center, Heidelberg, Germany.

${ }^{3}$ Protein Expression and Purification Core Facility, EMBL Heidelberg, Meyerhofstraße 1, Heidelberg, Germany

${ }^{4}$ Department of Molecular Biology, Princeton University, Princeton, New Jersey, USA

${ }^{5}$ Swiss Tropical and Public Health Institute, Basel CH-4002, Switzerland.

${ }^{6}$ University of Basel, Basel CH-4001, Switzerland.

*These authors contributed equally to this work.

†Correspondence to: e.stebbins@dkfz-heidelberg.de 
Suramin has been a primary early-stage treatment for African trypanosomiasis for nearly one hundred years. Recent studies revealed that trypanosome strains that express the Variant Surface Glycoprotein VSGsur possess heightened resistance to suramin. We show here that VSGsur binds tightly to suramin, other VSGs do not, and that together with VSG13 it defines a structurally divergent subgroup of these coat proteins. The co-crystal structure of VSGsur with suramin reveals that the chemically symmetric drug binds within a large cavity in the VSG homodimer asymmetrically, primarily through contacts of its central benzene rings. Structure-based, loss-of-contact mutations in VSGsur significantly decrease the affinity to suramin and lead to a loss of the resistance phenotype. Altogether, these data show that the resistance phenotype is dependent on the binding of suramin to VSGsur, establishing that the VSG proteins can possess functionality beyond their role in antigenic variation. 


\section{Introduction}

Multiple species of the genus Trypanosoma cause Sleeping Sickness in humans and related diseases in animals. These vector-borne illnesses are transmitted by the tsetse flies (Glossina spp.) and are primarily a burden in sub-Saharan Africa, impacting human populations directly through infection and indirectly through morbidity of livestock ${ }^{1,2}$. There are two subspecies that account for human infections: T. brucei gambiense (central and West Africa) and T. brucei rhodesiense (east and southern Africa). Numerous animal-pathogenic subspecies exist causing nagana in cattle (T. brucei brucei, T. congolense, T. vivax), surra in camels and horses (T. evansi, T. equiperdum), and trypanosomiasis in pigs and wild boars (T. simiae $)^{3}$.

A remarkable feature characterizing all such infections is the ability of the trypanosomes to thrive in the blood and tissue spaces of infected mammals despite complete exposure to the immune system of the host ${ }^{4}$. This is achieved largely through a dedicated machinery in the organism to alter its surface coat and continuously evade immune system recognition and clearance $^{5}$. The trypanosome surface is densely carpeted with approximately 10 million copies of the Variant Surface Glycoprotein (VSG), a GPI-anchored and glycosylated polypeptide to which the immune system mounts a very effective response ${ }^{6,7}$. The VSGs are long, rod-shaped homodimeric proteins of around 60kDa with two subdomains: a larger, N-terminal domain (NTD) of around 350-400 amino acids and a smaller 80-120 residue C-terminal domain (CTD) to which the GPI anchor is attached (Supplementary Fig. 1$)^{8}$. The NTDs consist of a conserved three-helix bundle scaffolding, the loops connecting the helices adorned with large insertions forming top and bottom subdomains or lobes (Supplementary Fig. 1). The top lobe (facing away from the parasite) is hypothesized to harbor the majority of immune epitopes, presenting the "antigenic face" of the 
VSG, although little epitope mapping and no antibody-VSG co-crystal structures have been published to date ${ }^{8-10}$.

While the host immune system develops rapid and effective responses to the VSGs, the trypanosomes access a genetic repository of over 2000 VSG genes and pseudo-VSG genes with distinct antigenic properties, switching coat proteins and thereby rendering the host response naïve to the new coat before the parasites can be cleared. This process of recognition, partial clearance, coat switching, and pathogen escape is termed antigenic variation. Because of this ability to thwart immune clearance, African trypanosomiasis is usually fatal unless treated chemotherapeutically ${ }^{4}$.

Synthesized as early as 1917, the compound suramin (originally Bayer 205 and later sold as Germanin) was first used therapeutically to treat African trypanosomiasis in the $1920 \mathrm{~s}^{11-13}$. It is one of only a few drugs (including pentamidine, melarsoprol, eflornithine, nifirtimox, and fexinidazole $)^{12,13}$ available to counter the disease and is only effective in the early stages of infection, before the parasite has entered the central nervous system (as suramin cannot effectively penetrate the blood-brain barrier $\left.{ }^{14}\right)$. It has also been used prophylactically ${ }^{15}$ and is on the World Health Organization's List of Essential Medicines ${ }^{16}$. Recently, suramin has demonstrated potent antineoplastic properties ${ }^{17}$. It is part of a larger family of benzopurpurine dyes and naphthalene ureas that show trypanocidal activity ${ }^{12}$. Resistance to suramin has gradually spread through the animal-infective trypanosomal populations, but has yet to reach those species causing disease in humans ${ }^{18}$.

The specific mechanism behind the trypanolytic activity of suramin remains unresolved, although studies have implicated effects on the glycosome and impairment of cytokinesis ${ }^{19,20}$. A model for the internalization of suramin has been proposed in which the drug enters through receptor-mediated endocytosis involving two distinct pathways: (1) low-density lipoproteins 
(LDLs) with possible involvement of other serum proteins and (2) the invariant surface glycoprotein 75 (ISG75) receptor of the pathogen ${ }^{21-23}$. However, to date no direct binding of suramin to any trypanosomal protein has been reported.

Recently, in vitro selection in the presence of suramin generated trypanosome strains that were over ninety-fold resistant to the drug ${ }^{18}$. All these resistant strains were shown to express a specific VSG, termed VSGsur, that itself was sufficient to convey the resistant phenotype in trypanosomes genetically engineered to express $\mathrm{it}^{23}$. However, it has remained unclear how VSGsur is involved in this resistance phenotype, whether directly or indirectly. Hypotheses include a model in which VSGsur causes suramin resistance by decreasing specific, receptor-mediated endocytosis pathways critical to the uptake of the drug ${ }^{23}$.

We show here that VSGsur directly binds suramin with high affinity, whereas other VSGs do not. Crystal structures of VSGsur and VSG13 identify a subfamily of VSGs that structurally diverge from all those resolved to date in several aspects, including a cavity between the monomers in the VSG-homodimer interface. The co-crystal structure of VSGsur with suramin shows that the drug binds in this cavity, while a structure-based, single loss-of-contact mutation abrogates both suramin binding and resistance. 


\section{Results}

\section{Structures of VSGsur and VSG13 reveal a divergent subfamily of VSGs}

We sought insight into the function of VSGsur through structural studies of the protein (clone from T. brucei rhodesiense, Genbank ATI14856), determining the crystal structure of the NTD to $1.2 \AA ̊$ resolution (residues 30-408, Fig. 1a, Methods, Supplementary Fig. 2 and Table 1). VSGsur differs markedly from previous VSG structures determined (VSG1, VSG2, VSG3, and ILTat1.24) ${ }^{24-27}$. While the core 3-helix bundle scaffolding of the VSG family is present, VSGsur possesses several divergent features. The most striking is observed in the "upper lobe" of the molecule. The insertion between the helices at the top of VSGsur folds into a bona fide structural subdomain (residues $\sim 140-260$ ) consisting of five $\beta$-strands and an elongated loop between 174208 that travels half the length of the VSG molecule before returning to the upper lobe (Supplementary Fig. 3a). This subdomain forms an intermolecular $\beta$-sandwich with the pairing monomer in the homodimer to create a tightly interwoven quaternary fold with a significant hydrophobic core. Perched on top of the 3-helix bundle, this "head" is strikingly different than the more flattened polypeptide arrangements of the other VSG top lobes that do not organize into any typified fold.

Also remarkable is the location of an N-linked sugar nearly two-thirds of the distance up the NTD from the bottom lobe and directly under the $\beta$-sandwich top lobe. Most VSGs possess Nlinked carbohydrates, but in all studied to date they are located in the bottom lobe and have been hypothesized to function there in VSG-membrane dynamics ${ }^{28}$. Further distinguishing VSGsur from previous structures, the multiple disulfide bonds stabilizing most VSG proteins (three pairs in VSGsur) are not clustered in the upper lobe, but extend into the 3-helix bundle. Finally, there is a large cavity in the homodimeric interface in VSGsur not found in other published VSG structures, 
located just beneath the unusually placed N-linked glycan. Altogether, VSGsur is a significant departure from previously studied VSGs while still maintaining the core scaffolding of this family.

Our additional studies show that VSGsur is not a unique fold but is in fact likely a member of a subfamily of VSGs. The $1.4 \AA$ resolution crystal structure of VSG13 (also named MITat1.13) reveals a second VSG protein with a top "head" consisting of a large $\beta$-sheet subdomain (residues 130-260) that forms an intermolecular $\beta$-sandwich in the homodimer (Fig. 1b, Methods, Supplementary Fig. 3 and Table 2). Even more striking than VSGsur, the cysteine disulfide bonds of VSG13 (four in this case) are spread throughout the length of the VSG and not clustered in the top lobe, in fact reaching down near the bottom lobe of the NTD. As with VSGsur, VSG13 harbors an N-linked glycan approaching the top of the molecule, positioned just under the $\beta$-sheet head and not in the bottom lobe. VSG13 also possesses an internal cavity between monomers in the homodimer. While smaller than that observed in VSGsur, it is still large enough to allow solvent into the cavity from the crystallization reagents. A protein structural alignment of the VSGsur and VSG13 monomers (Supplementary Fig. 3b) shows that the core 3-helix bundle aligns well and the N-linked sugars superpose very closely, whereas the $\beta$-sheet head in the upper lobes align poorly, evincing significant divergence. Comparing VSGsur and VSG13 with the "canonical" VSG2 structure shows that the $\beta$-sandwich subdomain gives more height to the NTDs of this subfamily than the other VSGs previously studied (Supplementary Fig. 3c), which could have significance for membrane dynamics and receptor-mediated endocytosis ${ }^{28}$.

Although VSG13 and VSGsur present marked divergences from previously reported VSG structures, the variability in these two Variant Surface Glycoproteins is housed within more generally conserved features that make them clear members of a protein superfamily. Most obvious is the core 3-helix bundle scaffolding that undergirds all the VSGs and VSG-related 
proteins such as Serum Resistance-Associated protein (SRA) ${ }^{29}$ and the haptoglobin-hemoglobin receptor $^{30}$. The divergent structures in the top and bottom lobes of the VSGs are basically different insertions between the loops connecting the central helices of the core of these rod-like molecules. Secondly, the proteins all evince a shared functionality in antigenic variation by presenting distinct molecular surfaces to the extracellular environment with extensive variation in amino acid sequence, charge and hydrophobic distributions, and topography (Fig. 1c).

\section{Resistance studies with suramin}

To examine whether the resistance mechanism associated with the expression of VSGsur involved direct protein-to-drug binding, we tested T. brucei rhodesiense VSGsur (expressed in $T$. brucei brucei) and a panel of other VSGs from T. brucei brucei Lister 427 (including VSG13) for their effect on suramin susceptibility and suramin binding (Fig. 2). Toxicity experiments demonstrated that, similarly to what has been shown for several VSGs from T. brucei rhodesiense and T. brucei brucei strain BS22 $1^{18,23}$, suramin killed all VSG expressing Lister 427 strains we examined (VSG2, VSG3, and VSG13), whereas those expressing VSGsur were resistant to 2540x higher concentrations of the drug (Fig. 2a), while showing the same growth rate in the presence of $0.7 \mu \mathrm{M}$ suramin as in the absence of the drug (doubling time $6.5 \mathrm{~h}$, Supplementary Fig. 4). Isothermal titration calorimetry (ITC) showed that while suramin did not bind to VSG2, VSG3, or VSG13, it bound with nanomolar affinity to VSGsur (Fig. 2b and Supplementary Fig. 5).

\section{Co-crystal structure of VSGsur with suramin}

The above data motivated efforts to co-crystallize suramin together with VSGsur. Soaking native crystals in high concentrations of suramin led to well-diffracting crystals into which the 
drug could be modeled (Methods, Supplementary Table 1). Interestingly, the chemically symmetric drug suramin bound at the dimerization interface asymmetrically (Fig. 3a). Suramin binds tightly in the large cavity between the two monomers of VSGsur, burying a surface area of approximately $700 \AA^{2}$ with more than one hundred atomic contacts characterized by both hydrogen bonding and non-bonding interactions. Suramin $\left(\mathrm{C}_{51} \mathrm{H}_{40} \mathrm{~N}_{6} \mathrm{O}_{23} \mathrm{~S}_{6}\right)$ is a symmetric, polysulphonated naphthylurea (Supplementary Fig. 6a). The compound is centered on the urea functional group $(\mathrm{NH}-\mathrm{CO}-\mathrm{NH})$. Extending from the urea moiety in each direction are a pair of benzene rings (thus a total of four in the molecule), each connected by an amide and finally linking to a terminal naphthalene decorated with three sulfonic acid groups (therefore, two naphthalenes and six sulfonic acids in total, Supplementary Fig. 6a). The rotation axis of the VSGsur dimer passes through one of the benzene rings connected to the urea group (Fig. 3a and Supplementary Fig. 6b). Thus, the central urea group of suramin binds just "off center" with respect to the dimer rotation axis. Three of the four benzene groups in the center of the suramin molecule bind in the middle of the dimer interface. These are well-ordered in the structure, with clear electron density and Bfactors lower than the average of the overall structure (protein, ligands, and solvent). The naphthalene rings, however, show weaker electron density and high B-factors, characteristic of more disorder or conformational flexibility. Their extension outside the cavity results in fewer contacts from VSGsur to stabilize their conformation.

Four amino acids from each monomer in the dimer contribute the bulk of protein contacts to the drug: H122, R125, H126, and R292 (Fig. 3, b and c). These positively charged residues are centered in the cavity of the dimer interface, burying the majority of surface area upon drug binding. These major contacts are buttressed with a set of minor interactions from residues L49, R119, A123, and A129, as well as from the N-linked glycan, several of these moieties only making 
contact from one protein monomer to some of the more distally positioned elements of suramin (such as the more poorly ordered sulfonic acids connected to the naphthalene rings, Fig. 3, c and d). The side chain nitrogen atoms from two arginine residues, R125 and R292, both make hydrogen-bonds with the sulfate oxygens of the most distal naphthalene moieties of suramin, as does H126. Other residues such as L49, R119, A123, and A129 primarily make close-approach, non-bonding contacts (Fig. 3d).

The interface centers on the imidazole rings of H122. In both monomers these residues make extensive contacts, stacking with two of the benzene rings of suramin (Fig. 3b). In the native protein structure, H122 has two different, partially occupied conformations in the monomer of VSGsur (Supplementary Fig. 7, a-c). One is very similar to the conformation seen in the suramin bound co-crystal structure whereas the other is a different rotamer, flipped away from this position. The presence of the drug locks the histidines in the stacking interaction with the suramin benzene rings, abolishing any evidence of the other possible conformation. The suramin benzene ring between the two rings contacted by H122, and which is centered in the dimer interface, interacts with VSGsur via backbone and sidechain contacts from R125 and H126. Interestingly, the benzene group of the compound used to phase the structure (5-amino-2,4,6-triiodoisophthalic acid, I3C or "Magic Triangle", Methods), occupies the same position as the suramin benzene groups, stacking similarly with H122 (Supplementary Fig. 7d, Supplementary Table 1).

A secondary suramin binding mode was also discovered in a minority of the crystals. In this mode, H122 adopts the other possible conformer seen in the native structure. This conformation we term "closed" for how it reduces the size of the cavity (Supplementary Fig. 7b). In the closed conformation soak with suramin, the drug cannot occupy the same position as in the open conformation due to steric clash. Consistent with this, we observe difference density of a size 
possibly consistent with a molecule like suramin but positioned differently in a more extended conformation. However, the density is too weak to model with confidence. We hypothesize that as in the native structure, H122 is able to adopt two conformations, but that the open conformation with the stacking of the histidine rings over the suramin benzene groups leads to the better ordered interaction and thus likely represents the preferred binding mode. The closed conformation still leaves room for suramin to occupy the cavity, however, and may represent either a minor energetic state or transition from solution to the most stable binding mode.

The N-linked sugar groups (the $\mathrm{N}$-acetylglucosamine (GlcNAc) rings, the $\beta$-mannose, and another mannose residue in the carbohydrate chain) make contacts to the sulfonic acids decorating the naphthalene rings (Fig. 3c). These contacts only occur for the sugar chain of one molecule, as the asymmetric positioning of suramin in the cavity leads the other naphthalene group to protrude too far from the carbohydrate to make effective contacts.

\section{Mutations in the suramin binding site abolish drug resistance}

Altogether, the ITC and structural results show that unlike other VSGs, even the related VSG13, VSGsur binds suramin specifically and with high affinity. To examine whether the resistance phenotype of cells expressing VSGsur is tied to this binding, we created mutants (Supplementary Fig. 8, Methods) that should not disrupt the fold of VSGsur. One mutant was a direct loss-of-contact alteration, changing H122 to alanine (H122A), thereby removing the stacking of the two histidine rings to the benzene groups in the most ordered binding mode of the ligand. The second was indirect, mutating N130 to alanine (N130A), thereby preventing the Nlinked glycosylation from occurring and removing the sugar-to-drug interactions. 
Fig. 2a summarizes these results. Both mutants were viable and grew in the absence of the drug, although the N130A mutant evinced a lower growth rate with a doubling time of $8.9 \mathrm{~h}$

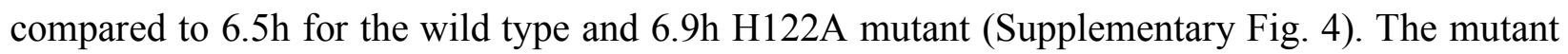
VSGs behaved similarly to the wild type protein during purification, suggesting that the changes did not destabilize or otherwise compromise the proteins. However, they both lacked resistance to suramin (Fig. 2a). This loss is correlated with a loss in binding to the drug (Fig. 2b), directly linking the suramin-VSGsur interaction to the resistance phenotype.

To verify that the mutations did not perturb the fold of VSGsur, we solved the crystal structure of the VSGsur mutant H122A (Supplementary Table 3), showing a nearly identical structure to wild type VSGsur. We were unable to obtain well-diffracting crystals of the mutant N130A, likely as the sugar is significantly involved in crystal packing contacts. High concentration, long-duration soaks of suramin into the H122A mutant (Methods, Supplementary Table 3), however, revealed the presence of significant difference density in the pocket, but the density was poorly ordered and we were unable to confidently model suramin (Supplementary Fig. 9).

\section{Distal alterations to the suramin binding pocket are associated with increased resistance to}

\section{suramin}

We further subjected T. $b$. rhodesiense STIB900_sur ${ }^{18}$, which expresses VSGsur and is about 100 -fold more resistant to suramin than other strains ( $\mathrm{IC}_{50}$ of $1.1 \pm 0.13 \mu \mathrm{M}$ ), to increasing suramin pressure in vitro (up to $18 \mu \mathrm{M}$ over one year). This produced "supermutants" that tolerated higher levels of the drug $\left(\mathrm{IC}_{50}\right.$ of $\left.11 \pm 1.2 \mu \mathrm{M}\right)$. Analysis of the expressed VSG of a highly resistant clone showed that it was VSGsur with 14 mutations in the nucleotide sequence, producing eight amino acid substitutions in the protein sequence. These mutations clustered in the structure in two 
locations (Supplementary Fig. 10). G174T, E175A, and K288A are located around the N-linked glycan with several contacts to the sugar. They do not, however, contact the drug but could conceivably modulate contacts to suramin indirectly through its interactions with the carbohydrate. A304S, T313A, D317E, A318T, and A326T cluster on one face of the bottom lobe, distal from the drug binding site, creating a patch of surface of exposed residues. This distance from the binding pocket makes it very likely that these mutations do not affect drug binding and thus could represent a functional region of VSGsur that interacts with other factors critical to generating resistance.

A construct of the mutant VSGsur was targeted into the active VSG expression site of $T . b$. rhodesiense STIB900_sur1 (Supplementary Figure 11a). Of four transfected clones, two had replaced VSGsur with the mutant version and two had retained the original VSGsur, as verified by PCR. The "pseudo-clones" with the original VSGsur sequence showed equivalent resistance to previously characterized strains expressing VSGsur, whereas the two clones that expressed the mutant version of VSGsur showed an increase in their $\mathrm{IC}_{50}$ to suramin (Supplementary Fig. 11b), suggesting that the "supermutant" mutations indeed further enhance suramin resistance. However, this increased resistance was only 2-3-fold higher than wild type VSGsur, indicating that there are likely other mutations in the selected supermutant strains that synergize with the VSGsur mutations to produce the full 11-fold increase in $\mathrm{IC}_{50}$.

\section{Alterations in endocytic trafficking are not coupled to resistance}

Wiedemar et al. had shown that VSGsur expressing strains have reduced levels of endocytic trafficking relative to VSG2 (also called VSG221), and, in particular, a reduced internalization of LDL, a factor hypothesized to be involved in suramin uptake into the cell ${ }^{21-23}$. Therefore, VSGsur 
might enhance resistance to the drug by decreasing suramin uptake through reducing endocytosis via the LDL receptor. However, as VSGsur likely comprises over $99 \%$ of the surface protein on the parasite and binds suramin with nanomolar affinity at blood $\mathrm{pH}$, understanding suramin uptake in these strains is complicated by the potential for a massive influx of the drug as the VSG is internalized and then recycled back to the membrane ${ }^{31}$. To interrogate this model, we performed endocytosis experiments with blue dextran and bodipy-labeled LDL (Fig. 4, Methods). Our data show that, consistent with previous results ${ }^{23}$, there is no generalized defect in trafficking in our VSGsur expressing strains (Fig. 4, a and b). In fact there appears to be a wide variation between different VSGs on the rates of trafficking (Fig. 4a). While we do observe that VSGsur cells have a significant decrease in LDL uptake compared to VSG2 (although, again, there is substantial variance among different VSGs, Supplementary Fig. 10), loss-of-binding suramin mutants show no significant alteration in these kinetics (Fig. 4c). Similar results are seen with uptake via the transferrin receptor (Fig. 4d). As these mutants also lose resistance to suramin, it seems that the dynamics of LDL uptake (and thus, presumably, suramin via this pathway) are not strongly coupled to the resistance phenotype in VSGsur. In contrast, the key determinant appears to be the binding of the drug to the VSG.

\section{Discussion}

Despite the wide genetic variance in the VSGs, only a handful of the membrane distal, large NTD domains have been characterized at the atomic level ${ }^{24-27}$. The recent crystal structure of VSG3 revealed topological divergence from the folds of other VSG NTDs, demonstrating that there is significantly more variation in architecture of this protein family than previously considered $^{25}$. This structure also uncovered an unexpected post-translational modification $(O-$ 
linked sugar at the top of VSG3) that strongly modulates the host immune response. With the structures of VSGsur and VSG13 presented here, we show that the "antigenic space" of the VSGs is much broader than anticipated even from the VSG3 results. Moreover, the co-crystal structure of VSGsur with the trypanocidal compound suramin directly links the binding of the drug to the resistance phenotype displayed by strains of $T$. brucei expressing VSGsur.

This binding of suramin establishes that the VSGs can have a function beyond that of antigenic variation. This idea is buttressed by recent results that show that VSG2 is able to bind a ligand as well (the divalent metal calcium, manuscript in preparation). Also consistent with this, the recently determined crystal structure of the $T$. congolense haptoglobin-hemoglobin receptor ${ }^{30}$ shows that this protein adopts a fold very similar and clearly related to the VSGs, packing functionally with all possible VSGs on the surface as it is required for parasite survival in the host to scavenge iron. These observations raise the possibility of other functions for the VSGs in binding small molecules or even proteins, and to therefore function for the benefit of the pathogen in manners more diverse than mere immune evasion.

We are not aware of any data showing that suramin-resistant field isolates express VSGsur. VSGsur was identified in the laboratory under cell culture conditions of increasing suramin concentrations, where there is no pressure to switch to alternative VSGs that can prevent immune clearance (in contrast to the situation in the host). If VSGsur undergirds resistance in the context of infection, antigenic variation and VSG switching would need to be modeled. Wiedemar et al. ${ }^{18}$ noted that short-term survival under low-dose suramin treatment in animals could allow for the development of alternative mechanisms of drug resistance. Another model would involve the display of multiple, antigenically distinct versions of VSGsur. This could conceivably occur through gene duplication and divergence of surface features while preserving the suramin binding 
cavity or through the formation of mosaics that switch CTDs in a manner that alters the antigenicity of the NTDs (as has been recently reported ${ }^{32}$ ).

While the binding of suramin to VSGsur is seen to be required for drug resistance, it is still unclear by what mechanism VSGsur is producing this effect. Genetic studies coupled with examination of the trypanosome's single lysosome suggest that suramin accumulates in this compartment, while other evidence links suramin toxicity to effects on the glycosome and impairment of cytokinesis ${ }^{19,20}$. Our internalization data decouple suramin uptake via the LDL endocytic pathway from the VSGsur-related mechanism of resistance. This focuses attention on the interaction of the drug with VSGsur itself. However, it is unclear how to link resistance to (presumably) lysosomal trafficking and downstream toxicity to the VSGsur-suramin interaction. The simplest model involves VSGsur acting as a cellular repository or "sponge" that reduces the trafficking of the drug, shunting it to some kind of disposal pathway and thereby reducing the effective concentration. Since VSGsur will be produced at high levels in the trypanosome and exported toward the cell surface from the Golgi through the vesicular trafficking pathways, it is conceivable that suramin that is internalized through other pathways (e.g., via the LDL receptor and ISG75) could comingle with VSGsur in specific endocytic compartments, the latter binding the drug and preventing it from trafficking further (Fig. 5). Such a disposal pathway could involve shuttling the drug from the vesicular trafficking pathway to the extracellular space or another compartment that renders it inactive.

The VSGsur "supermutants" could offer a tantalizing clue toward solving this puzzle and uncovering the mechanism. While one subset of the mutations clusters around the carbohydrate and could conceivably alter binding to the drug, another set clusters in a surface patch on the bottom lobe of the protein. This patch is suggestive of a binding site and may point the way toward 
the macromolecular interactions involved in suramin resistance. Future experiments will likely require a tracking of the drug through the trypanosome to establish how VSGsur binding modulates toxicity. The results of this study focus such investigations squarely on the VSG as the key player in resistance, and also provide numerous tools (such as structure based mutants) to interrogate the biochemical process allowing strains to survive in high concentrations of suramin. 


\section{Methods}

\section{Cloning and Production of $T$. brucei strains}

A VSG13 expressing T. brucei clone was produced as described ${ }^{33}$ and was kindly provided by the group of Luisa Figueiredo (Universidade de Lisboa). VSGSur from T. brucei rhodesiense (GenBank: MF093647.1) was codon-optimized and synthesized as a pUC19 clone (BioCat, Germany), and introduced into T. brucei strain Lister427 as described in Supplementary Methods. VSGsur mutants N130A and H122A were generated by site-directed mutagenesis using the QuikChange Lightening kit (Agilent Technologies) according to the manufacturer's protocol. Transfections were performed into a $T$. brucei brucei cell line expressing VSG2, termed $2 \mathrm{~T} 1^{34} .5$ 10 micrograms of purified plasmids were mixed with $100 \mu 13 \times 10^{7}$ cells in Tb-BSF buffer ${ }^{35}$ (90 mM Phosphate buffer, $\mathrm{pH}$ 7.3, $5 \mathrm{mM} \mathrm{KCl,} 50 \mathrm{mM}$ HEPES, $\mathrm{pH}$ 7.3, $0.15 \mathrm{mM} \mathrm{CaCl2}$ ) and electroporated using a Lonza Nucleofector 2b, program Z-001 or X-001. After 8 hours of incubation hygromycin B was added to a concentration of 5-25 $\mu \mathrm{g} \mathrm{ml}^{-1}$. Single cell clones were obtained by serial dilutions in 24 well plates in standard culture medium and screened by FACS for VSG2-antibody binding. VSG2-antibody negative clones (positive transfectants were identified by lack of binding) were sequenced: RNA was isolated using the RNeasy Mini Kit including an on-column DNase treatment (Qiagen). Complementary DNA was synthesized with SuperScript IV Reverse Transcriptase (Invitrogen) according to the manufacturer's protocol. Amplification was performed with a forward primer binding the spliced leader sequence and a reverse primer binding in the VSG 3'UTR using Phusion high fidelity DNA polymerase. PCR products were purified by gel extraction from a 1\% agarose gel, followed by the NucleoSpin Gel and PCR clean up kit (Macherey-Nagel) and sent to Eurofins (Ebersberg) for sequencing using the same primers as for the PCR. 


\section{Purification of VSGs}

T. b. brucei expressing VSG2, VSG3, VSG13, VSGsur, VSGsur-N130A, and VSGsurH122A mutants were cultivated in vitro in HMI-9 media (formulated as described by PAN Biotech without FBS, L-cysteine, or ß-mercaptoethanol), supplemented with 10\% fetal calf serum (Gibco), L-cysteine, and $\beta$-mercaptoethanol. Cells were cultured at $37^{\circ} \mathrm{C}$ and $5 \% \mathrm{CO}_{2}$. VSGs were purified according to established protocols ${ }^{37}$. Briefly, cells were pelleted and then lysed in $0.4 \mathrm{mM} \mathrm{ZnCl}_{2}$. The lysis mixture was centrifuged and the pellet containing the membrane material was resuspended in pre-warmed $\left(40{ }^{\circ} \mathrm{C}\right) 10 \mathrm{mM}$ phosphate buffer, $\mathrm{pH}$ 8. Following a second centrifugation, supernatant containing VSG protein was loaded onto an anion-exchange column (Q-Sepharose Fast-Flow, GE Healthcare), which had been equilibrated with $10 \mathrm{mM}$ phosphate buffer, $\mathrm{pH}$ 8. The flow-through containing highly pure VSG was concentrated in an Amicon Stirred Cell and optionally shock frozen in liquid nitrogen and stored at $-80^{\circ} \mathrm{C}$.

For crystallization of VSG13, the C-terminal domain was removed by digestion with Endoproteinase LysC (NEB) at a 1:800 LysC:substrate ratio by mass for $1-2 \mathrm{~h}$ at $37^{\circ} \mathrm{C}$. VSG13 Nterminal domain was purified by gel filtration on a HiLoad 16/600 Superdex 200pg column (GE Healthcare) in $50 \mathrm{mM}$ HEPES, pH 7.6, $150 \mathrm{mM} \mathrm{NaCl}$. Lysine residues were methylated (as per ${ }^{38}$ ), using Dimethyl-amine-borane complex (Sigma) and Formaldehyde (Thermo, Methanol-free). The methylated VSG13 N-terminal domain was purified by gel filtration on a Superdex 200 Increase 10/300 GL (GE Healthcare) in $10 \mathrm{mM}$ Tris.Cl, pH 8.0. Fractions from the gel filtration runs were subjected to SDS-PAGE analysis for visual inspection.

For crystallization of VSGsur and VSGsur mutants, the C-terminal domain was removed by limited proteolysis with trypsin at a 1:100 trypsin:substrate ratio by mass for $1 \mathrm{~h}$ on ice. The 
VSGsur N-terminal domain was purified by gel filtration on a HiLoad 16/600 Superdex 200pg column (GE Healthcare) in $10 \mathrm{mM}$ Tris.Cl, $\mathrm{pH}$ 8.0. The peak fractions were pooled and concentrated to $4 \mathrm{mg} / \mathrm{ml}$.

\section{Structural Determination of VSG13}

Purified methylated VSG13 N-terminal domain was concentrated to $2.5 \mathrm{mg} \mathrm{ml}^{-1}$ in $10 \mathrm{mM}$ Tris. $\mathrm{Cl} \mathrm{pH} \mathrm{8.0.} \mathrm{Crystals} \mathrm{were} \mathrm{grown} \mathrm{at} 23^{\circ} \mathrm{C}$ by vapour diffusion using hanging drops formed from mixing a 1:1 volume ratio of the protein with an equilibration buffer consisting of 1.8-2.0 M $\left(\mathrm{NH}_{4}\right)_{2} \mathrm{SO}_{4}, 100 \mathrm{mM}$ Tris. $\mathrm{Cl} \mathrm{pH}$ 8.5. For cryoprotection, crystals were transferred into the same buffer including $20 \%$ glycerol and flash-cooled immediately afterward to $100 \mathrm{~K}\left(-173.15^{\circ} \mathrm{C}\right)$. For phase determination, crystals were soaked for $30 \mathrm{~s}$ in a buffer containing $0.5 \mathrm{M}$ sodium bromine and $20 \%$ glycerol before flash-freezing.

Native data were collected at the European Synchrotron Radiation Facility (ESRF) at a wavelength of $1 \AA \AA$ on beamline ID29 and bromine soaks at the Diamond Light Source at $0.9198 \AA$ on beamline i03 (Supplementary Table 2). The data were phased by single-wavelength anomalous diffraction (SAD) from 33 bromine sites that were identified using the SHELX suite ${ }^{39}$. Several models were manually combined from automated model building by PHENIX ${ }^{40}$ and CRANK of $\mathrm{CCP}^{41}$ into maps produced from the SHELX sites and improved by several cycles of manual building, auto-building (PHENIX), and refinement (PHENIX) into the native dataset (Supplementary Table 2). 


\section{Structural Determination of VSGsur and VSGsur mutants}

Crystals were grown at $4^{\circ} \mathrm{C}$ by vapour diffusion using hanging drops by mixing $2 \mu 1$ of the protein solution $(4 \mathrm{mg} / \mathrm{ml})$ with $2 \mu \mathrm{l}$ of the equilibration buffer $(19-24 \%$ PEG 400, $100 \mathrm{mM}$ $\mathrm{TEA} / \mathrm{HCl} \mathrm{pH}=7.5$ and $10 \%(\mathrm{v} / \mathrm{v})$ isopropanol). The crystals were transferred to a cryobuffer $(40 \%$ PEG400 and $100 \mathrm{mM} \mathrm{TEA} / \mathrm{HCl}$ ) and flash-cooled in liquid nitrogen. Native datasets were collected at a wavelength of $0.9184 \AA$ at the Helmholtz-Zentrum Berlin at Beamline MX 14.1. For phasing the crystals were soaked overnight in $50 \mathrm{mM}$ 5-amino-2,4,6-triiodoisophthalic acid (I3C)/LiOH (Magic Triangle, Jena Biosciences) ${ }^{42}$ in cryobuffer. The I3C soaked crystals were collected at a wavelength of $2.066 \AA$ at the Helmholtz-Zentrum Berlin at Beamline MX 14.2. The structure was solved by SAD from the anomalous signal from two I3C molecules bound per VSGsur monomer using the SHELX ${ }^{39}$ and the HKL3000 ${ }^{43}$ suite. Arp/wARP 44 was used for automated building of the initial model. PHENIX ${ }^{40}$ and COOT $^{45}$ were used for several cycles of model building and refinement.

Suramin complexes were obtained by either soaking VSGsur crystals in the drug or by premixing and growing crystals de novo. Complexes were produced by soaking the crystals between 1 hour and 6 days in cryobuffer supplemented with 0.77 - $7.7 \mathrm{mM}$ Suramin before cryo-cooling. Co-crystals were obtained at $4^{\circ} \mathrm{C}$ using $3 \mathrm{mg} / \mathrm{ml}$ VSGsur, $0.7 \mathrm{mM}$ Suramin and an equilibration buffer containing 16-20\% PEG 3350, $200 \mathrm{mM} \mathrm{NaCL}$ and $100 \mathrm{mM}$ Hepes/ $\mathrm{NaOH} \mathrm{pH}=7.5$. Prior to cryo-cooling these crystals were transferred to $16 \%$ PEG 3350, $200 \mathrm{mM} \mathrm{NaCl}, 100 \mathrm{mM}$ Hepes/NaOH pH=7.5, $0.7 \mathrm{mM}$ Suramin and $25 \%$ PEG 400. The VSGsur-suramin datasets were collected at the Helmhotz-Zentrum Berlin, the Paul Scherrer Institut Villingen, and Diamond Light Source. The structures were solved by molecular replacement with PHENIX using the native structure of VSGsur as the search model (Supplementary Table 1). 
The incubation of native crystals with suramin produced an unexpected effect on the crystal packing. Native VSGsur crystallized in the space group $\mathrm{P} 2{ }_{1} 2{ }_{1} 2$ with a single molecule in the asymmetric unit and a two-fold axis of crystallographic symmetry aligned with the dimerization axis, thereby producing a crystallographic dimer highly similar to that observed in other VSGs. One hour soaks of native crystals in $0.7 \mathrm{mM}$ and $7.7 \mathrm{mM}$ suramin did not change these parameters and led to structures where additional difference density appeared in the cavity but which could not be modeled. However, as the soak time increased (greater than 4 hours), a shift in the crystallographic symmetry was observed and correlated with increased evidence of electron density for suramin. In particular, the two-fold rotational symmetry no longer aligned with the dimerization axis of rotation, causing a shift in the contents of the crystal to harbor two molecules (a single homodimer of VSGsur) in the asymmetric unit in a lower symmetry space group. These changes were tolerated by the crystals, maintaining high diffraction. In these soaks, electron density for suramin was clear and able to be modeled.

\section{Uptake Assays}

The LDL and transferrin uptake assays were performed as described ${ }^{23}$ with slight modifications while the dextran assays were performed similarly. Cells were cultured to a density of approximately $1 \times 10^{6}$ cells per mL of HMI-9. For LDL and transferrin, the cells were pelleted and washed in serum free medium containing $1 \%$ BSA before being returned to $37^{\circ} \mathrm{C}$ for 15 minutes to remove any surface-bound ligand. Approximately 100,000 cells were aliquoted into assay tubes in duplicate and kept at $37^{\circ} \mathrm{C}$. Pre-warmed bodipy-labeled LDL (Invitrogen; ThermoFisher catalog number I34359) suspended in serum free medium containing 1\% BSA was added to the tubes at the indicated time points such that the final volume of the assay was $150 \mu \mathrm{L}$ and the concentration 
of LDL was $15 \mu \mathrm{g} / \mathrm{mL}$. Being at a much higher concentration as a stock, $2 \mu \mathrm{L}$ of transferrin (Invitrogen; ThermoFisher catalog number T13342) was added to the assay tubes to a final volume of $200 \mu \mathrm{L}$ and a final concentration of $50 \mu \mathrm{g} / \mathrm{mL}$. The assays were conducted such that all conditions reached the end point of the assay simultaneously. At time zero, all vials were placed on ice and $1 \mathrm{~mL}$ of ice cold serum free medium containing 1\% BSA was added to the tubes. The cells were washed twice by centrifugation at $2000 \times \mathrm{G}$ for 5 minutes and resuspended in ice cold serum free medium containing 1\% BSA. The vials were then kept on ice until being analyzed on a Becton Dickinson FACSCalibur. Fluorescence intensity was quantified by analysis in FlowJo after gating on cells determined by forward and side scatter. The geometric mean of fluorescence was then calculated for between 7000 and 10,000 cells per replicate per time point for each cell line assayed. To control for differences in intrinsic auto-fluorescence, each cell line was individually normalized to its own "time 0 " point, which was collected from cells that had been exposed to ice cold ligand and immediately placed on ice. These "time 0" points were generally within only 1-2 fluorescence units of each other. For dextran, approximately 100,000 cells were aliquoted into assay tubes and pelleted. The cells were suspended in $50 \mu \mathrm{L}$ of $0.5 \mathrm{mg} / \mathrm{mL}$ Alexa 488-dextran diluted in HMI-9 and kept at $37^{\circ} \mathrm{C}$ until reaching the expiry of the incubation. Once reaching those time points, the cells were immediately placed on ice and $1 \mathrm{~mL}$ of ice cold HMI-9 was added to the tubes. The cells were washed three times by centrifugation at $2000 \times \mathrm{x}$ for 5 minutes and resuspended in ice cold HMI-9. The vials were then kept on ice until being analyzed on a Becton Dickinson FACSCalibur and fluorescence was quantified as above.

\section{Production of "Supermutants"}


Bloodstream-forms of T. b. rhodesiense_sur1 were further selected in vitro by stepwise increases of the suramin concentration from $1 \mu \mathrm{M}$ to $18 \mu \mathrm{M}$ over the course of one year. The expressed VSG gene was amplified from cDNA by PCR with primers binding to the 5' spliced leader and a conserved sequence (gatatatttaaca) on the $3^{\prime}$ untranslated region of VSGs ${ }^{46,47}$. For transfection, a construct was used containing the blasticidine resistance gene and the coding sequence of mutant VSGsur separated by a $\alpha \beta$ tubulin splice site, and framed with the 5' and 3' UTRs of VSGsur (Supplementary Fig. 11). Transfectant pseudoclones were generated by limiting dilution as described $^{18}$.

\section{Determination of growth rates and drug sensitivity assays}

Cumulative growth curves for different VSG-expressing T. brucei strains were determined by dilution of the cells to $10^{5} \mathrm{ml}^{-1}$ in $25 \mathrm{~cm}^{2}$ cell culture flasks in duplicate. After incubation with $0.7 \mu \mathrm{M}$ suramin for $24 \mathrm{~h}$ the cell densities were determined by cell counting using a Neubauer hemocytometer. This procedure was repeated for 3-4 days in a row. Analysis was performed with GraphPad Prism, using a nonlinear regression model for curve fitting (Exponential growth with $\log )$.

For the drug sensitivity assays, serial dilutions of suramin (Sigma, dissolved in water and stored in aliquots at $-20^{\circ} \mathrm{C}$ ) as well as blank cultures without suramin, were prepared in 24 well plates (at least 3 technical replicates). Cells were added to a density of $10^{5} \mathrm{ml}^{-1}$. After $24 \mathrm{~h}$ the cell densities were determined with a Neubauer hemocytometer. The 50\% Inhibitory concentrations $\left(\mathrm{IC}_{50}\right)$ were calculated with GraphPad Prism, using the non-linear regression model to fit the dose-response curve (variable slope; four parameters). 


\section{Isothermal Titration Calorimetry (ITC)}

ITC experiments were performed using a PEAQ ITC (Malvern) at $20^{\circ} \mathrm{C}$. Titration buffers contained $10 \mathrm{mM} \mathrm{NaPi}(\mathrm{pH} 8.00)$ and $150 \mathrm{mM} \mathrm{NaCl}$. Proteins were transferred into the titration buffer by gel filtration chromatography, followed by concentration in 10k disposable ultrafiltration centrifugal devices. Protein concentrations were measured by UV absorbance at $280 \mathrm{~nm}$. In each experiment, the protein concentration in the cell varied between $40-55 \mu \mathrm{M}$. Suramin was injected at concentrations between 300 and $600 \mu \mathrm{M}$, and all samples were degassed prior to each experiment. All VSG protein-suramin experiments were performed at least in duplicate to check the reproducibility of the data. The data were baseline corrected, integrated, and analyzed with the PEAQ ITC Analysis software (Malvern), fitted using a single-site binding model.

\section{Data Availability}

Coordinates and structure factors have been uploaded to the RCSB PDB (www.rcsb.org): VSGsur I3C (PDB ID 6Z79), VSGsur WT native (PDB ID 6Z7A), VSGsur + Suramin (PDB ID 6Z7B), VSGsur H122A (PDB ID 6Z7C), VSGsur H122A 0.77mM Suramin (PDB ID 6Z7D), VSGsur H122A 7.7 mM (PDB ID 6Z7E), VSG13 NaBr (PDB ID 6Z8G, and VSG13 native (PDB ID $6 \mathrm{Z} 8 \mathrm{H})$. 


\section{REFERENCES}

1. Ponte-Sucre, A. An Overview of Trypanosoma brucei Infections: An Intense Host-Parasite Interaction. Front. Microbiol. 7, (2016).

2. Keating, J., Yukich, J. O., Sutherland, C. S., Woods, G. \& Tediosi, F. Human African trypanosomiasis prevention, treatment and control costs: A systematic review. Acta Trop. 150, 4-13 (2015).

3. Radwanska, M., Vereecke, N., Deleeuw, V., Pinto, J. \& Magez, S. Salivarian Trypanosomosis: A Review of Parasites Involved, Their Global Distribution and Their Interaction With the Innate and Adaptive Mammalian Host Immune System. Front. Immunol. 9, 2253 (2018).

4. Matthews, K. R., McCulloch, R. \& Morrison, L. J. The within-host dynamics of African trypanosome infections. Philos Trans R Soc Lond B Biol Sci 370, (2015).

5. Mugnier, M. R., Stebbins, C. E. \& Papavasiliou, F. N. Masters of Disguise: Antigenic Variation and the VSG Coat in Trypanosoma brucei. PLoS Pathog 12, e1005784 (2016).

6. Cross, G. A. Identification, purification and properties of clone-specific glycoprotein antigens constituting the surface coat of Trypanosoma brucei. Parasitology 71, 393-417 (1975).

7. Overath, P. \& Engstler, M. Endocytosis, membrane recycling and sorting of GPI-anchored proteins: Trypanosoma brucei as a model system. Mol Microbiol 53, 735-44 (2004).

8. Aresta-Branco, F., Erben, E., Papavasiliou, F. N. \& Stebbins, C. E. Mechanistic Similarities between Antigenic Variation and Antibody Diversification during Trypanosoma brucei Infection. Trends Parasitol. 35, 302-315 (2019). 
9. Bangs, J. D. Evolution of Antigenic Variation in African Trypanosomes: Variant Surface Glycoprotein Expression, Structure, and Function. BioEssays 40, 1800181 (2018).

10. Carrington, M. \& Higgins, M. K. O -h what a surprise. Nat. Microbiol. 3, 856-857 (2018).

11. Schnitzer, R. J. \& Hawking, F. Experimental Chemotherapy. (Elsevier, 2013).

12. Steverding, D. The development of drugs for treatment of sleeping sickness: a historical review. Parasit. Vectors 3, 15 (2010).

13. Lindner, A. K. et al. New WHO guidelines for treatment of gambiense human African trypanosomiasis including fexinidazole: substantial changes for clinical practice. Lancet Infect. Dis. 20, e38-e46 (2020).

14. Sanderson, L., Khan, A. \& Thomas, S. Distribution of Suramin, an Antitrypanosomal Drug, across the Blood-Brain and Blood-Cerebrospinal Fluid Interfaces in Wild-Type and PGlycoprotein Transporter-Deficient Mice. Antimicrob. Agents Chemother. 51, 3136-3146 (2007).

15. Gill, B. S. \& Malhotra, M. N. Prophylactic Activity of Suramin Complexes in 'Surra' ( Trypanosoma evansi ). Nature 200, 285-286 (1963).

16. WHO | WHO Model Lists of Essential Medicines. WHO http://www.who.int/medicines/publications/essentialmedicines/en/.

17. Stein, C. A. Suramin: a novel antineoplastic agent with multiple potential mechanisms of action. Cancer Res. 53, 2239-2248 (1993).

18. Wiedemar, N. et al. Beyond immune escape: a variant surface glycoprotein causes suramin resistance in Trypanosoma brucei: Suramin resistance in T . brucei. Mol. Microbiol. 107, 5767 (2018). 
19. Babokhov, P., Sanyaolu, A. O., Oyibo, W. A., Fagbenro-Beyioku, A. F. \& Iriemenam, N. C. A current analysis of chemotherapy strategies for the treatment of human African trypanosomiasis. Pathog. Glob. Health 107, 242-252 (2013).

20. Thomas, J. A. et al. Insights into antitrypanosomal drug mode-of-action from cytology-based profiling. PLoS Negl. Trop. Dis. 12, e0006980 (2018).

21. Alsford, S. et al. High-throughput decoding of antitrypanosomal drug efficacy and resistance. Nature 482, 232-236 (2012).

22. The uptake of the trypanocidal drug suramin in combination with low-density lipoproteins by Trypanosoma brucei and its possible mode of action. - PubMed - NCBI. https://www.ncbi.nlm.nih.gov/pubmed/7902661.

23. Wiedemar, N. et al. Expression of a specific variant surface glycoprotein has a major impact on suramin sensitivity and endocytosis in Trypanosoma brucei. FASEB BioAdvances 1, 595608 (2019).

24. Bartossek, T. et al. Structural basis for the shielding function of the dynamic trypanosome variant surface glycoprotein coat. Nat Microbiol 2, 1523-1532 (2017).

25. Pinger, J. et al. African trypanosomes evade immune clearance by O-glycosylation of the VSG surface coat. Nat. Microbiol. 3, 932-938 (2018).

26. Freymann, D. et al. 2.9 A resolution structure of the N-terminal domain of a variant surface glycoprotein from Trypanosoma brucei. J. Mol. Biol. 216, 141-160 (1990).

27. Metcalf, P., Blum, M., Freymann, D., Turner, M. \& Wiley, D. C. Two variant surface glycoproteins of Trypanosoma brucei of different sequence classes have similar $6 \mathrm{~A}$ resolution X-ray structures. Nature 325, 84-6 (1987). 
28. Hartel, A. J. et al. N-glycosylation enables high lateral mobility of GPI-anchored proteins at a molecular crowding threshold. Nat Commun 7, 12870 (2016).

29. The structure of serum resistance-associated protein and its implications for human African trypanosomiasis | Nature Microbiology. https://www.nature.com/articles/s41564-017-00853?WT.mc_id=COM_NMicro_1801_Higgins.

30. Higgins, M. K. et al. Structure of the trypanosome haptoglobin-hemoglobin receptor and implications for nutrient uptake and innate immunity. Proc. Natl. Acad. Sci. 110, 1905-1910 (2013).

31. Engstler, M. et al. Kinetics of endocytosis and recycling of the GPI-anchored variant surface glycoprotein in Trypanosoma brucei. J Cell Sci 117, 1105-15 (2004).

32. Jayaraman, S. et al. Application of long read sequencing to determine expressed antigen diversity in Trypanosoma brucei infections. PLoS Negl. Trop. Dis. 13, e0007262 (2019).

33. Figueiredo, L. M., Janzen, C. J. \& Cross, G. A. M. A Histone Methyltransferase Modulates Antigenic Variation in African Trypanosomes. PLOS Biol. 6, e161 (2008).

34. Alsford, S. \& Horn, D. Single-locus targeting constructs for reliable regulated RNAi and transgene expression in Trypanosoma brucei. Mol. Biochem. Parasitol. 161, (2008).

35. Schumann Burkard, G., Jutzi, P. \& Roditi, I. Genome-wide RNAi screens in bloodstream form trypanosomes identify drug transporters. Mol. Biochem. Parasitol. 175, 91-94 (2011).

36. Hirumi, H. \& Hirumi, K. Continuous cultivation of Trypanosoma brucei blood stream forms in a medium containing a low concentration of serum protein without feeder cell layers. $J$ Parasitol 75, 985-9 (1989).

37. Cross, G. A. Release and purification of Trypanosoma brucei variant surface glycoprotein. $J$ Cell Biochem 24, 79-90 (1984). 
38. Rypniewski, W. R., Holden, H. M. \& Rayment, I. Structural consequences of reductive methylation of lysine residues in hen egg white lysozyme: An x-ray analysis at 1.8-.ANG. resolution. Biochemistry 32, 9851-9858 (1993).

39. Sheldrick, G. M. A short history of SHELX. Acta Crystallogr A 64, 112-22 (2008).

40. Adams, P. D. et al. PHENIX: a comprehensive Python-based system for macromolecular structure solution. Acta Crystallogr Biol Crystallogr 66, 213-21 (2010).

41. Winn, M. D. et al. Overview of the CCP4 suite and current developments. Acta Crystallogr. D Biol. Crystallogr. 67, 235-242 (2011).

42. Beck, T., Krasauskas, A., Gruene, T. \& Sheldrick, G. M. A magic triangle for experimental phasing of macromolecules. Acta Crystallogr. D Biol. Crystallogr. 64, 1179-1182 (2008).

43. Minor, W., Cymborowski, M., Otwinowski, Z. \& Chruszcz, M. HKL-3000: the integration of data reduction and structure solution - from diffraction images to an initial model in minutes. Acta Crystallogr. D Biol. Crystallogr. 62, 859-866 (2006).

44. Langer, G., Cohen, S. X., Lamzin, V. S. \& Perrakis, A. Automated macromolecular model building for X-ray crystallography using ARP/wARP version 7. Nat Protoc 3, 1171-9 (2008).

45. Emsley, P., Lohkamp, B., Scott, W. G. \& Cowtan, K. Features and development of Coot. Acta Crystallogr. D Biol. Crystallogr. 66, 486-501 (2010).

46. Aline, R. et al. (TAA)n within sequences flanking several intrachromosomal variant surface glycoprotein genes in Trypanosoma brucei. Nucleic Acids Res. 13, 3161-3177 (1985).

47. Cross, G. A. M., Kim, H.-S. \& Wickstead, B. Capturing the variant surface glycoprotein repertoire (the VSGnome) of Trypanosoma brucei Lister 427. Mol. Biochem. Parasitol. 195, 59-73 (2014). 
48. Eisenberg, D., Schwarz, E., Komaromy, M. \& Wall, R. Analysis of membrane and surface protein sequences with the hydrophobic moment plot. J. Mol. Biol. 179, 125-142 (1984).

49. Kelley, L. A., Mezulis, S., Yates, C. M., Wass, M. N. \& Sternberg, M. J. The Phyre2 web portal for protein modeling, prediction and analysis. Nat Protoc 10, 845-58 (2015).

50. McNicholas, S., Potterton, E., Wilson, K. S. \& Noble, M. E. M. Presenting your structures: the CCP4mg molecular-graphics software. Acta Crystallogr. D Biol. Crystallogr. 67, 386394 (2011).

51. Schrodinger. The PyMOL Molecular Graphics System, Version 1.8. (2015).

52. de Beer, T. A. P., Berka, K., Thornton, J. M. \& Laskowski, R. A. PDBsum additions. Nucleic Acids Res. 42, D292-D296 (2014).

53. LigPlot+: Multiple Ligand-Protein Interaction Diagrams for Drug Discovery | Journal of Chemical Information and Modeling. https://pubs.acs.org/doi/10.1021/ci200227u.

\section{SUPPLEMENTARY FIG.S AND TABLES}

Figures 1-12

Table 1-3

\section{ACKNOWLEDGEMENTS}

We acknowledge time at the European Synchrotron Radiation Facility (ESRF, beamline ID29, proposal MX1975, Gianluca Santoni and colleagues), the Diamond Light Source (DLS, beamline i03, proposal number mx18989-1, Neil Paterson and colleagues), the Helmholtz Zentrum Berlin (BESSY, beamline 14.1 and 14.2, proposal number MX-191-00036 and MX-192-00114, staff member Manfred Weiss and colleagues, specially Christian Feiler for the help with processing and solving the I3C SAD dataset), and the Paul Scherrer Institut, Villingen, Switzerland (SLS, 
beamline X06DA, proposal number 20182345, 20191097 and 20191895, Vincent Olieric and colleagues). We thank Luisa Figueiredo for supplying us with the VSG13 expressing T. brucei strain.

\section{AUTHOR INFORMATION}

H.H. cloned VSGsur and M.vS. produced VSGsur mutants. S.H., N.W., and P.M. produced VSGsur "supermutant" clones and assays. M.vS. and J.Z. purified all VSGs. M.sV. crystallized VSG13. M.vS. and J.Z. crystallized wild type and mutant VSGsur proteins. C.E.S. phased and produced the first model of VSG13 and A.H. built and refined the structures of VSG13 native and the sodium bromide soak. J.Z. solved all crystal structures of VSGsur. P.J. worked with J.Z. on the refinement of VSGsur bound to suramin. M.vS. performed the suramin growth and toxicity assays. K.P. conducted ITC experiments. J.V. conducted endocytosis assays. 


\section{FIG. LEGENDS}

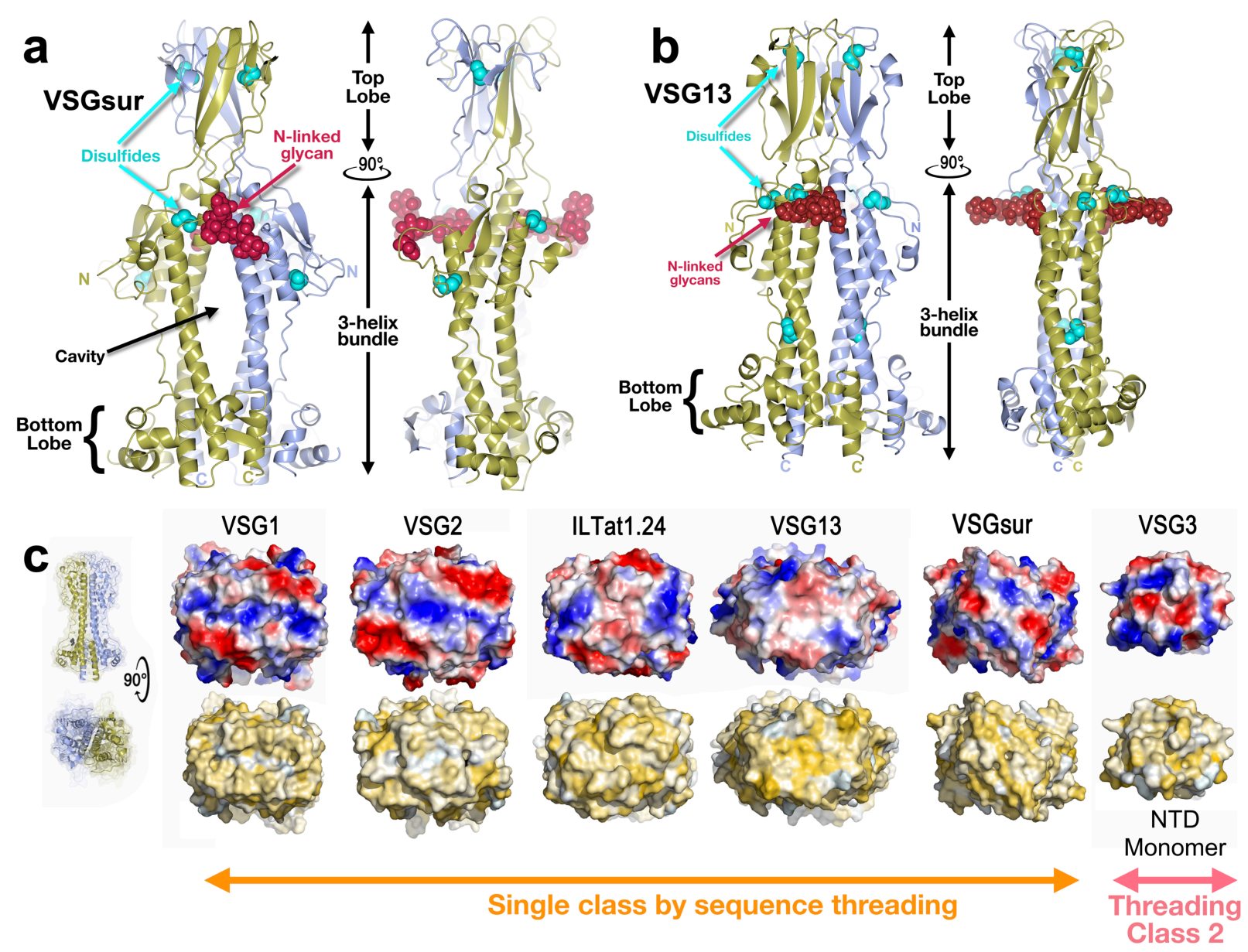

Fig. 1: Overall Structure of VSGsur.

(a) VSGsur is shown as a ribbon diagram in two orientations related by a 90 degree rotation, the monomers in the homodimer colored gold and light blue. The N-linked glycans of the two VSG monomers are displayed as red space-filling atoms, disulfide bonds are shown in cyan, and several key features are labeled (top and bottom lobes, 3-helix bundle, and the cavity between the dimers. " $\mathrm{N}$ " and "C" indicate the N- and C- termini of each monomer, color coded to match the ribbon diagrams. (b) The crystal structure of VSG13 in two orientations is depicted similarly to that of VSGsur as in (a). (c) Molecular surfaces of published VSG structures to date. The VSGs are 
oriented looking "down" on the top lobe of the protein, the Fig. rotated 90 degrees about a horizontal axis in comparison to previous Fig.s. The upper row of surfaces is colored by relative electrostatic potential (blue is basic/positively charged, red is acidic/negatively charged, and white is neutral) and on the lower row by the Eisenberg hydrophobicity scale ${ }^{48}$ (yellow indicates hydrophobic, white polar). Arrows underneath the surfaces illustrate the two broad superfamilies of different VSGs identified by sequence threading as implemented by PHYRE2 ${ }^{49}$. Note that the NTD of VSG3 is monomer in solution and the crystal structure, and in the absence of any structural data of a NTD dimer, we have chosen to show only the monomeric surface. Structures and molecular surfaces illustrated with CCP4mg ${ }^{50}$ and MacPyMOL ${ }^{51}$. 


\section{Cell growth with suramin $24 \mathrm{~h}$}

a

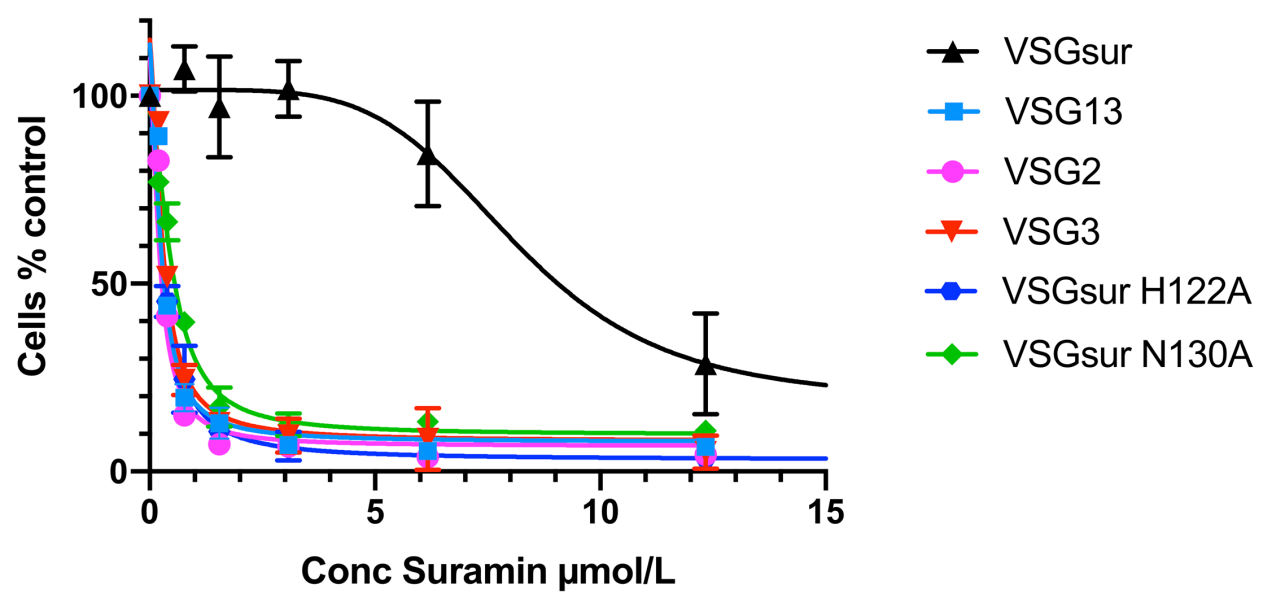

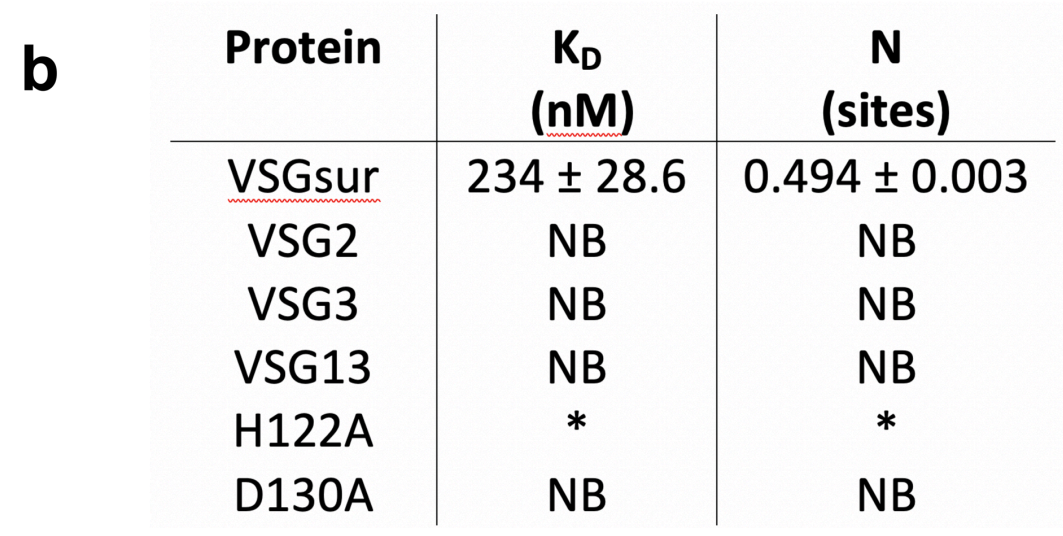

Fig. 2: Suramin Binds only VSGsur and is toxic to all other VSGs examined

(a) Growth curves at different suramin concentrations show that VSG2, VSG3, and VSG13 are sensitive to the drug and only VSGsur shows resistance, whereas two structure-based mutants in VSGsur (H122A and N130A) lose this resistance. ( $\mathrm{n}=3$ sampled). (b) Isothermal calorimetric results of suramin binding to different VSGs. Matching the toxicity assays, suramin was found to bind tightly only to wild type VSGsur, but not well to the two structure-based mutants (H122A and N130A) or VSG2, VSG3, and VSG13. "NB" indicates no binding. “*” for the VSGsur H122A mutant indicates evidence of weak binding (Supplementary Fig. 5), 
bioRxiv preprint doi: https://doi.org/10.1101/2020.06.04.134106; this version posted June 5, 2020. The copyright holder for this preprint (which was not certified by peer review) is the author/funder, who has granted bioRxiv a license to display the preprint in perpetuity. It is made available under aCC-BY-NC-ND 4.0 International license.

but a satisfactory fit to the curve was not possible. The binding for $\mathrm{H} 122 \mathrm{~A}$ is expected to be in the high $\mu \mathrm{M}$ range. 


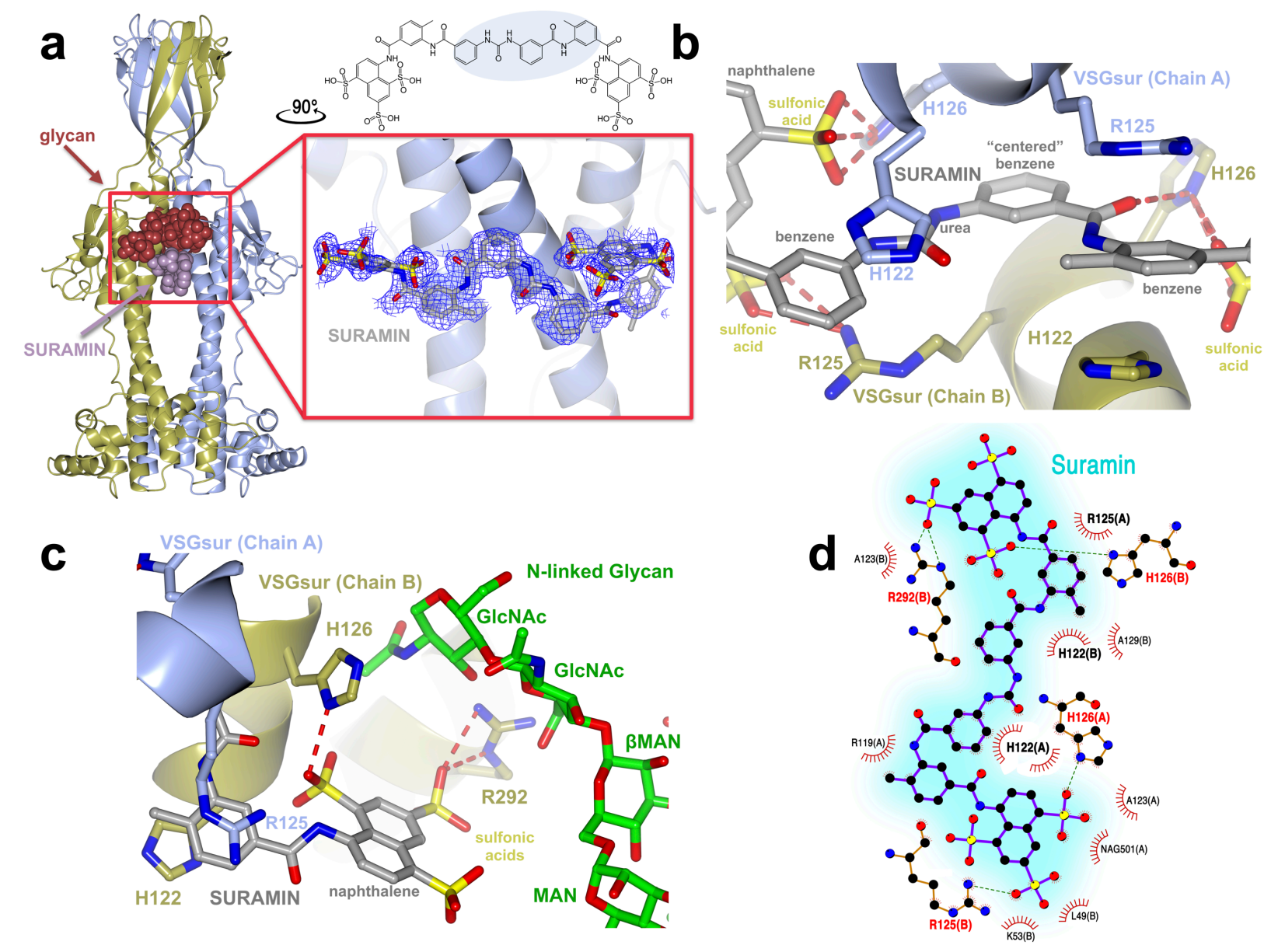

Fig. 3: Suramin binds in the cavity between the VSGsur monomers

(a) On the left is the overall structure of the VSGsur-suramin co-crystal structure in ribbon diagram form similar to that depicted in Fig. 1. Suramin is shown in purple as a space-filling representation. The red box around the drug is expanded in the boxed image on the right (rotated 90 degrees counterclockwise about a vertical axis), showing a ball-and-stick rendering of the compound with a blue wire-cage of the $1 \sigma$ contour of the $1.9 \AA 2$ Fo-Fc electron density map of the refined molecule drawn around it. Suramin is shown as a ball-and-stick chemical representation with oxygen atoms are shown in red, nitrogen in blue, and carbon gray. (b) VSGsur is depicted as in Fig. 1 and suramin as in panel (a). Side-chains from VSGsur are shown in ball-and-stick representation and colored according to the chain to which they belong (blue and gold). Hydrogen bonds are shown as red- 
dashed lines. (c) Contacts between the N-linked glycan (with carbon atoms colored green, oxygen red, and nitrogen blue) and VSGsur, shown and colored as in (b). GlcNAc indicates NAcetylglucosamine, MAN indicates mannose, and $\beta$ MAN indicates $\beta$-mannose. (d) Schematic illustrating the contacts of VSGsur to suramin, focused on the ligand (generated by PDBsum ${ }^{52}$ using LigPlot ${ }^{53}$ ). Structures were illustrated with CCP4mg ${ }^{48}$. 

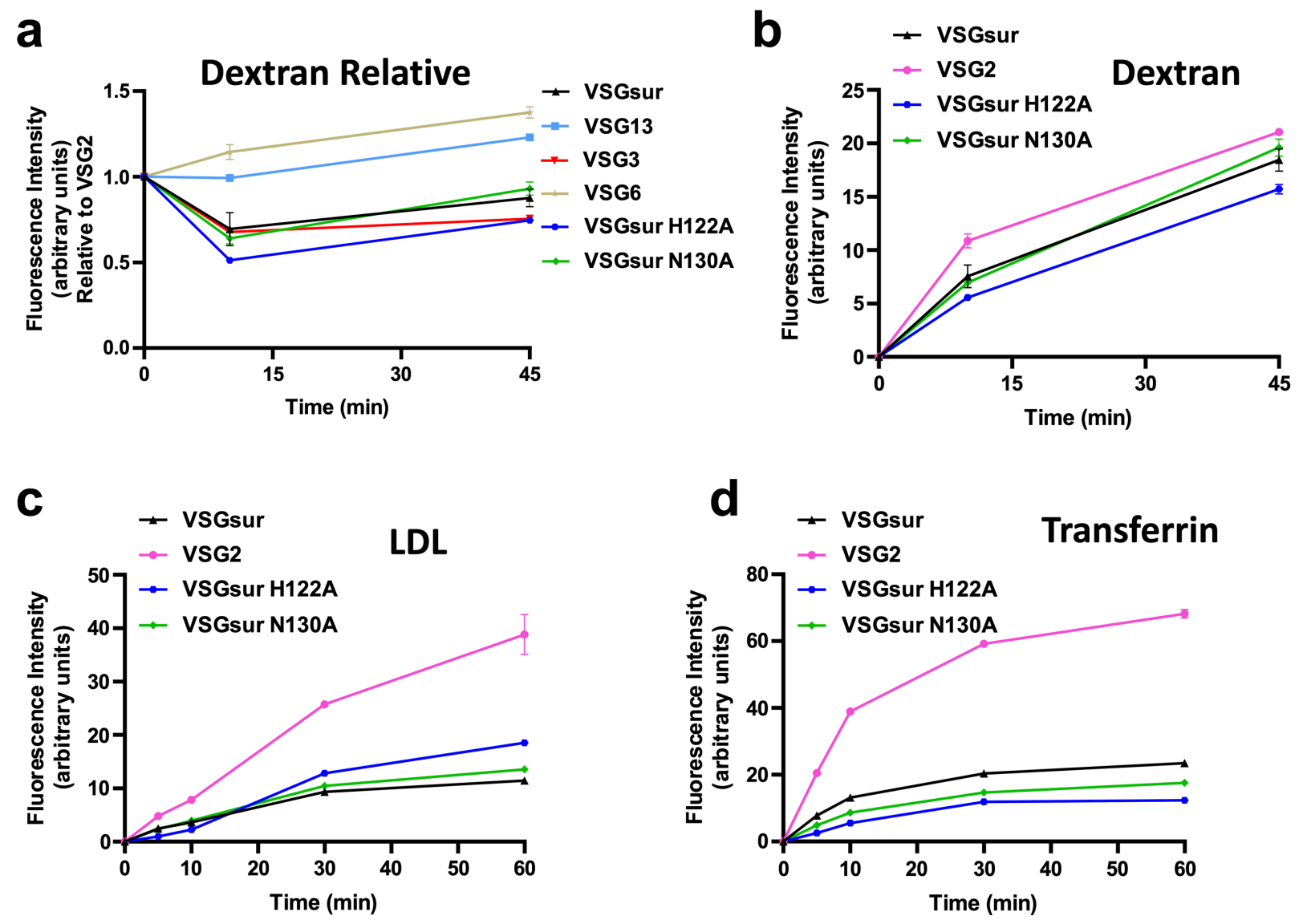

Fig. 4: VSGsur and the loss of contact mutants have indistinguishable endocytic kinetics.

(a) Alexa 488-dextran endocytosis by T. b. brucei $2 \mathrm{~T} 1$ cells expressing a variety of different VSG genes. Due to technical limitations, each cell line could not be analyzed simultaneously within each experiment for each ligand. Therefore, VSG2 was used as a control in all experiments, allowing the calculation of the relative uptake rate for each cell line rate as compared to the uptake rate of each ligand observed by VSG2 expressing cells within each separate experiment. Each of these graphs therefore represent the combined experimental results from 2 to 3 separate experiments. Cell lines with a relative fluorescence intensity below 1 at a given time point have less efficient endocytic rates compared to VSG2 expressing cells, and vice versa. All graphs share the same $\mathrm{Y}$ axis. Error bars when present denote the range $(\mathrm{N}=2$ experimental replicates for each 
cell line at every time point), while error bars were left out when they were smaller than the size of the data point and therefore not visible. (b)-(d) Alexa 488-dextran (b), bodipy-LDL (c), and Alexa 488-transferrin (d) endocytosis by T. b. brucei $2 \mathrm{~T} 1$ cells expressing VSGsur (black triangles), VSG2 (pink circles), VSGsur H122A (blue hexagons), or VSGsur N130A (green diamonds). All graphs depict arbitrary fluorescence units, determined by flow cytometry, on the $\mathrm{Y}$ axis. Each graph represents one of multiple biological replicates of each experiment, with $\mathrm{N}=2$ experimental replicates for each time point. Error bars when present denote the range, while error bars were left out when they were smaller than the size of the data point and therefore not visible. 


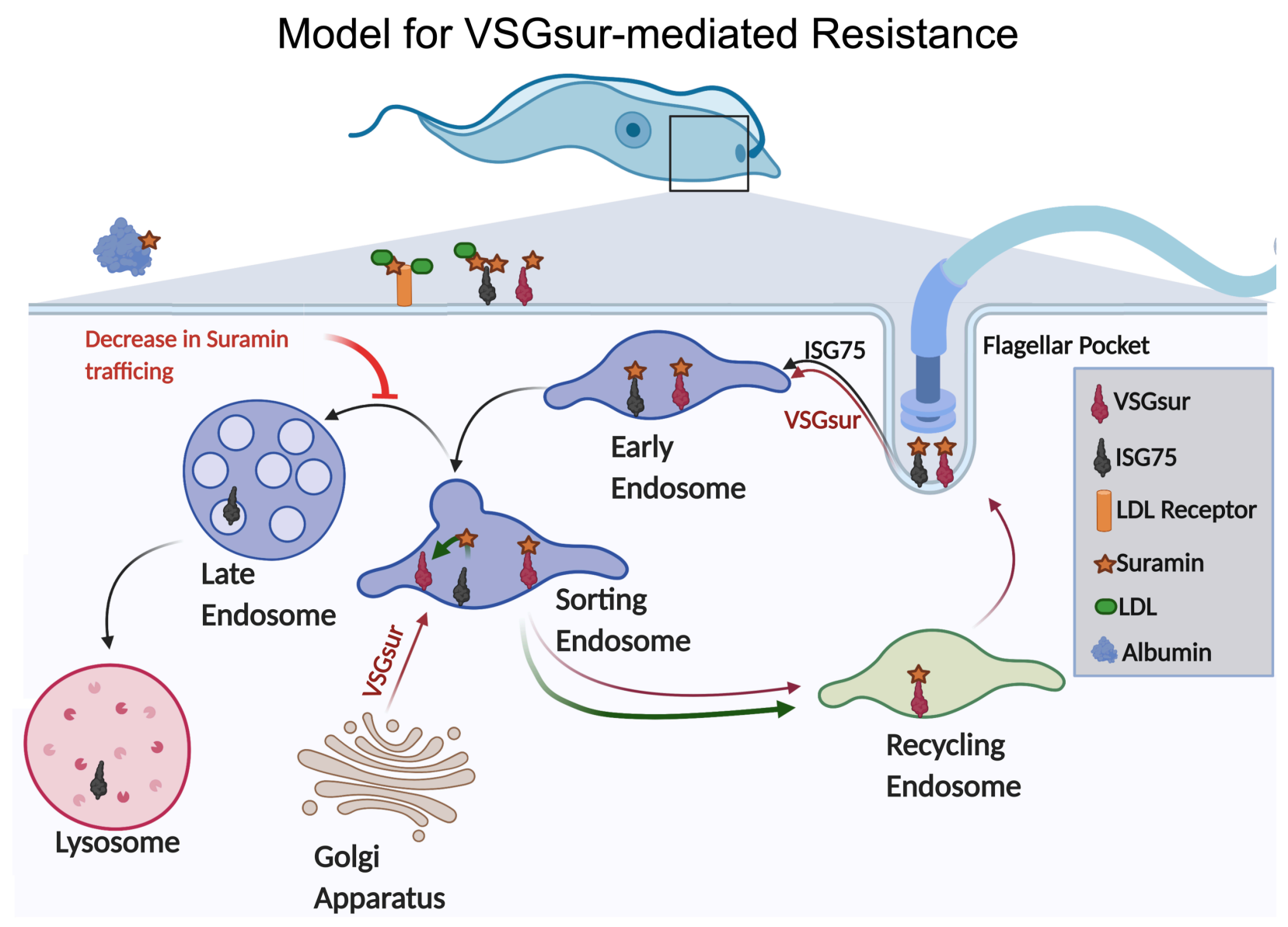

Fig. 5: Model for possible mechanism of VSGsur-mediated resistance to suramin. A schematic illustration of how VSGsur could shuttle suramin away from toxic endocytic internalization pathways. A single trypanosome cell is depicted in blue at the top of the diagram, the boxed region magnified showing the vesicular transport compartments. Critical players like ISG75, the LDL receptor, blood proteins, and VSGsur are shown as defined in the key. In this model, VSGsur acts as a "sponge" to remove suramin from the endocytic pathway, decreasing its effective concentration and thereby rendering the cell more resistant to the drug. 